\title{
Combating HIV resistance - focus on darunavir
}

\author{
Cécile L Tremblay' \\ 'Department of Microbiology and \\ Immunology, Centre Hospitalier de \\ l'Université de Montréal, Montréal, \\ Canada
}

\begin{abstract}
Darunavir is a second-generation protease inhibitor designed to have antiviral efficacy against HIV-1 isolates harboring multiple resistance mutations to protease inhibitors. Pivotal trials conducted in treatment-experienced HIV-infected individuals have demonstrated significantly greater virological suppression when darunavir was added to an optimized background treatment compared with a control protease inhibitor. This virological suppression was associated with an increase in CD4 counts and was sustained over time. Darunavir resistance-associated mutations have been defined as V11I, V32I, L33F, I47V, I50V, I54L/M, G73S, L76V, I84V, and L89V. In clinical trials, baseline darunavir susceptibility was a strong predictor of virological response. Prior use of fosamprenavir was associated with darunavir resistance mutations. Darunavir has a high genetic barrier and has a distinct resistance profile. Although some cross-resistance exists with other second-generation protease inhibitors such as tipranavir, different resistance mutation patterns have been observed upon failure to these regimens. It was found that mutations at $47 \mathrm{~V}, 54 \mathrm{M}, 85 \mathrm{~V}$, and $73 \mathrm{~T}$ were most prevalent in isolates resistant to both PIs. Mutations $48 \mathrm{~V}, 50 \mathrm{~V}$, and 54L were associated with resistance to darunavir but not to tipranavir. $82 \mathrm{~S}$ and $82 \mathrm{~T}$ were associated with resistance to tipranavir but not to darunavir. Therefore, darunavir provides potent virological efficacy as well as high genetic barrier that can be useful to preserve treatment options in HIV-infected, treatmentexperienced individuals.
\end{abstract}

Keywords: resistance, antiretroviral therapy, protease inhibitor, HIV-1

\section{Introduction}

Antiretroviral drug resistance has become a major challenge in designing treatment regimens for HIV-infected individuals, particularly those who have been exposed to serial monotherapy or dual-therapy before the advent of using combination antiretroviral (ARV) therapy that included non-nucleoside reverse transcriptase inhibitors (NNRTI) or protease inhibitors (PI) in the mid 1990s. In a cohort of 4306 patients having started ARV therapy with 2 nucleoside analogs and a third agent, the risk of developing resistance over 6 years was $27 \%$. The prevalence of resistance to 2 and 3 classes of ARVs in this chronically infected population was estimated to be $20 \%$ and $5 \%$, respectively (Kuritzkes 2007). The development of new ARV agents active against drug-resistant isolates has brought new perspective on the treatment of HIV-infected, treatment-experienced individuals. Guidelines for the treatment of HIV-infected individuals, such as those developed by the International Aids SocietyUSA (IAS-USA) or the US Department of Health and Human Services (US DHHS), recommend that achieving plasma viral loads $<50$ copies $/ \mathrm{mL}$ should be the goal for all treated individuals, including highly treatment-experienced ones (Hammer et al 2006; DHHS 2008). Pivotal studies that led to these recommendations include the TORO, RESIST, and POWER trials that studied enfuvirtide, ritonavir-boosted tipranavir, and darunavir, respectively. Here the role of darunavir in our therapeutic armamentarium is reviewed. 


\section{Darunavir pre-clinical development and in vitro resistance studies}

Darunavir exhibits potent HIV antiviral activity in vitro with a $50 \%$ effective concentration (EC50) of 1 to $5 \mathrm{nM}$ and a $90 \%$ effective concentration of 2.7 to $13 \mathrm{nM}$ (De Meyer et al 2005). Darunavir is well absorbed, and its bioavailability increases by $30 \%$ when given with food. Darunavir is approximately $95 \%$ bound to plasma proteins. It is metabolized by and inhibits cytochrome P-450 (CYP) isoenzyme 3A4; therefore, darunavir is prone to CYP3A4-mediated drug - drug interactions (Surleraux et al 2005; Busse and Penzak 2007).

\section{Mechanisms}

Darunavir is a second-generation PI specifically designed to be active against PI-resistant HIV-1 isolates. HIV protease (PR) cleaves large gag-pol polyproteins into smaller proteins such as p24, p17, and p7, p6, p2, and p1, that are necessary for HIV assembly and RNA packaging resulting in infectious virions (Flexner 1998). PIs inhibit this enzyme by binding to its active site. Structure-based design of HIV-1 PIs that can specifically interact with the enzyme backbone atoms has led to stereochemically defined bis(tetrahydrofuranyl)(THF) urethane as a non-peptidic P2 ligand (Ghosh et al 2007). This bis-THF group confers strong hydrogen binding to the active site of the enzyme. Its binding affinity for the wildtype enzyme is 2 orders of magnitude higher than that of first-generation PI and maintains 1.5 orders of magnitude tighter binding to the multi-drug resistant enzyme (King et al 2004). Structural analyses revealed that the close contact of darunavir with the main chains of the PR active-site amino acids (Asp-29 and Asp-30) is important for its potency and wide spectrum of activity against multi-PI-resistant HIV-1 variants (Koh et al 2003). Furthermore, because of its molecular flexibility, it can adapt itself to the changing shape of a mutant PR (Ghosh et al 2006; Tie et al 2007). Using ultra-high resolution crystal structure analysis of the darunavir complex with PR containing the drug-resistant mutation V32I and M46L, it was shown that darunavir binds at two distinct sites, one in the active-site cavity and the second on the surface of one of the flexible flaps in the PR dimer (Kovalevsky et al 2006a). Further studies of PR variants containing mutations D30N, I50V, and L90M demonstrated various inhibition constants reflecting different molecular interactions between the mutated PR and darunavir at each of these sites (Kovalevsky et al 2006b). It has also been shown that darunavir can block PR dimerization (Koh et al 2007).
Pre-clinical studies have shown darunavir's efficacy against a panel of 19 recombinant clinical isolates carrying multiple PR mutations and demonstrating resistance to an average of 5 other PIs. It was also active against a majority of 1500 PI-resistant recombinant viruses derived from clinical samples. In vitro selection of mutants resistant to darunavir starting from wild-type virus has proved to be extremely difficult (Surleraux et al 2005). After 75 passages (260 days), mutations at R41T and K70E appeared, resulting in approximately 10-fold phenotypic resistance. However, when the selected mutations were introduced into a laboratory strain by site-directed mutagenesis, they had no effect on susceptibility to darunavir or other PIs (De Meyer et al 2005). When this experiment was extended to 327 passages (1155 days), mutations developed at H69Q and V77I, with an additional 8 mutations in the gag gene (both inside and outside the cleavage site), further decreasing phenotypic susceptibility.

\section{Clinical studies}

\section{Highly treatment-experienced patients}

An initial Phase IIa randomized, open-label, controlled study was conducted at 15 sites in Europe with 50 HIV-1-infected patients who had taken multiple PIs. PIs in non-suppressive regimens were replaced with darunavir/ritonavir (r) (300/100 or $600 / 100 \mathrm{mg}$ twice daily, or $900 / 100 \mathrm{mg}$ once daily), or left unchanged, for 14 days. Viral load responses in all darunavir/r groups (range, -0.56 to $-0.81 \log 10$ copies $/ \mathrm{mL}$ ) were greater $(\mathrm{p}<0.001)$ than in the controls $(-0.03 \log 10$ copies/mL). HIV-1 RNA $<400$ copies/mL at any time during treatment was achieved by $40 \%$ in the darunavir/r groups and $8 \%$ in the control group (Arasteh et al 2005). This study showed substantial antiviral activity of darunavir/r and led to 2 Phase IIb studies, POWER 1 and POWER 2. Both studies were designed to address treatment strategies in highly treatment-experienced individuals but were conducted in different geographical areas. Baseline mean viral loads were 4.66 and $4.48 \log _{10} \mathrm{c} / \mathrm{mL}$ and median CD 4 counts were 106 and 179 cells $/ \mu \mathrm{L}$ for POWER 1 and 2, respectively (Katlama et al 2007).

In these studies, after 24-week dose-finding phases and efficacy analyses, subjects continued on an optimized background regimen plus either darunavir/r 600/100 mg twice daily or a control PI. Combined data showed that 67 of 110 (61\%) darunavir/r treated subjects compared with 18 of $120(15 \%)$ of control subjects had viral load reductions of $1 \log _{10}$ copies/mL or greater from baseline (primary endpoint; difference in response rates $46 \%, 95 \%$ confidence interval 
[CI] 35\%-57\%, p $<0.0001)$. Based on a logistic regression model including stratification factors (baseline number of primary PI mutations, use of enfuvirtide, baseline viral load) as covariates, the difference in response was $50 \%$ (odds ratio $11.72,95 \%$ CI 5.75-23.89). A mean CD4 increase of 102 cells $/ \mathrm{mm}^{3}$ was observed in the darunavir $/ \mathrm{r}$ arms vs 19 cells $/ \mathrm{mm}^{3}$ in the comparator arms. In the darunavir $/ \mathrm{r}$ groups, rates of adverse events were mostly lower than or similar to those in the control groups, when corrected for treatment exposure (Clotet et al 2007). These impressive results in the Phase IIb studies at 48 weeks led to US FDA approval of darunavir in 2006.

Additional safety data were obtained in the POWER 3 trial (Molina et al 2007). Treatment-experienced HIV-1infected subjects received darunavir/ $\mathrm{r}$ at a dose of $600 / 100$ mg twice daily plus an optimized background regimen. Subjects treated numbered 327; the baseline mean HIV-1 RNA was $4.6 \log _{10}$ copies $/ \mathrm{mL}$, and the median CD4 count was 115 cells $/ \mathrm{mm}^{3}$ (median primary PI mutations $=3$, PI resistance-associated mutations $=9$ ). By the cutoff date 246 subjects reached week 24 and were included in the efficacy analysis: $65 \%$ and $40 \%$ achieved HIV-1 RNA reductions of $\leq 1 \log _{10}$ and $<50$ copies $/ \mathrm{mL}$, respectively, at week 24 . The mean CD 4 count increase was 80 cells $/ \mathrm{mm}^{3}$. The most common adverse events were diarrhea (14\%), nasopharyngitis $(11 \%)$, and nausea (10\%). These results corroborated those of POWER 1 and POWER 2. In all treatment-experienced clinical trials, darunavir has been relatively well tolerated. Few cases of hepatotoxicity have been observed in the postmarketing surveillance program (monography).

In POWER 1, 2, and 3, 11 mutations in the PR enzyme were associated with decreased responses to darunavir (V11I, V32I, L33F, I47V, I50V, I54L/M, G73S, L76V, I84V, and L89V). Baseline darunavir susceptibility was a strong predictor of virological response (Mascolini et al 2007). A 10-fold or less change in baseline susceptibility to darunavir resulted in a decrease in viral load of $2.08 \log _{10}$ copies $/ \mathrm{mL}$ at 24 weeks, whereas a 10- to 40 -fold change resulted in a decrease of $1.08 \log _{10}$ copies $/ \mathrm{mL}$ and a 40 -fold change, $0.76 \log _{10}$ copies/ $\mathrm{mL}$. The proportion of subjects achieving $<50$ copies $/ \mathrm{mL}$ of HIV RNA was 50\%, 25\%, and 13\% respectively. Mutations had varying impact on phenotypic susceptibility. Mutations V11I, I54L, G73S, and L89V were associated with a 2-fold decrease in susceptibility; V32I, L33F, and I47V, 2- to 3fold; I54M, L76V, and I84V, 3- to 4-fold; I50V, greater than 4-fold. The number of subjects achieving undetectable viral load was correlated with the number of mutations at baseline: $64 \%$ with no mutations, $50 \%, 42 \%, 22 \%$, and $10 \%$ with 1 ,
2, 3, and 4 mutations respectively (De Meyer et al 2006, 2008; Vangeneugden 2006; Kuritzkes 2007). Recent trials evaluated a new NNRTI, etravirine, in addition to a treatment regimen containing darunavir, in a treatment-experienced population. These DUET trials showed that when TMC-125 (etravirine) was added to an optimized background containing darunavir/ $\mathrm{r}$, there was an added benefit in the number of subjects reaching plasma viral loads $<50$ copies $/ \mathrm{mL}$ ( $56 \%$ vs $39 \%$ in the placebo arm) as well as in the number of individuals with more than one log decrease in viral load (Lazzarin et al 2007; Madruga et al 2007b).

\section{Moderately experienced patients}

Darunavir/r was compared with lopinavir/ $\mathrm{r}$ in a moderately treatment-experienced population in the TITAN trial (Madruga et al 2007a). In this trial, patients received an optimised background regimen plus non-blinded treatment with darunavir/r 600/100 mg twice daily or lopinavir/r 400/100 mg twice daily. Of 595 subjects randomized and treated, 187 (31\%) were PI-naïve; 476 of 582 (82\%) were susceptible to 4 or more PIs. At study entry, median phenotypic sensitivity score for the background regimen was 2.0 in both study arms, and trial participants had a median (range) of 0 (0 to 6) primary PI mutations, 4 ( 0 to 17 ) primary or other PI mutations, 0 ( 0 to 5 ) darunavir-related mutations, 1 ( 0 to 11 ) lopinavir-related mutations, and 2 ( 0 to 8 ) nucleoside-related mutations. Median (range) fold change in susceptibility was 0.6 ( 0.1 to 43.8$)$ for darunavir and 0.75 (0.3 to 74.5$)$ for lopinavir. At week 48, more darunavir/ $\mathrm{r}$ than lopinavir/ $\mathrm{r}$ patients had plasma HIV RNA of less than 400 copies $/ \mathrm{mL}$ (77\% [220 of 286] vs 68\% [199 of 293]; estimated difference $9 \%, 95 \%$ CI 2-16), meeting the primary end-point of non-inferiority ( $95 \%$ CI lower limit for the difference in treatment response $-12 \%$ or greater). Furthermore, in the ITT analysis, the lower limit of the CI (2\%) did not include $0 \%$, thereby meeting the a priori criterion for superiority. This was especially true in patients previously exposed to PI whose viruses harbored some resistance to PI. For subjects with viruses fully susceptible to protease inhibitors, both drugs performed similarly.

Darunavir resistance-associated mutations (RAMs) have been defined as V11I, V32I, L33F, I47V, I50V, I54L/M, G73S, L76V, I84V, and L89V. Lopinavir RAMS have been defined as L10F/I/R/V, K20M/R, L24I, V32I, L33F, M46I/ L, I47V/A, I50V, F53L, I54V/L/A/M/T/S, L63P, A71V/T, G73S, V82A/F/T/S, I84V, and L90M (Johnson et al 2007). The frequency of darunavir RAMs in this study was low. $83 \%$ of patients' baseline isolates had no darunavir RAMs. 
Only $4 \%$ of patients' baseline isolates had $\geq 3$ darunavir RAMs. A diminished response to darunavir/r was observed in patients with $\geq 3$ darunavir RAMs at baseline; this subgroup with $\geq 3$ darunavir RAMs at baseline had a median number of 13 RAMs. The virological response to lopinavir/ $\mathrm{r}$ was reduced (response $<75 \%$ of the overall response) in patients with 2 darunavir RAMs or with $\geq 6$ lopinavir RAMs at baseline. The presence of $\geq 6$ lopinavir RAMs at baseline had no influence on response to darunavir/r; this subgroup had a median number of 12 RAMs (Johnson et al 2007). Fewer subjects with virological failures (VF) who were treated with darunavir/r developed primary protease inhibitor mutations $(21 \%[\mathrm{n}=6]$ vs $36 \%[\mathrm{n}=20])$ and nucleoside analogue-associated mutations $(14 \%[n=4]$ vs $27 \%[n=15])$ than those treated with lopinavir/r. After excluding patients with lopinavir FC $>10$, the proportion of patients with VF developing primary PI mutations or NRTI RAMs was still higher in the lopinavir/r arm than the darunavir / $\mathrm{r}$ arm (33\% vs $4 \%$, and $23 \%$ vs $13 \%$, respectively). Fewer patients with VF on darunavir/r than on LPV/r lost susceptibility compared with baseline to the PI or an NRTI used in the treatment regimen. The V32I mutation developed in $>10 \%$ of patients with VF $(3 / 28 ; 11 \%)$ on darunavir / $\mathrm{r}$ treatment. In this treatment-experienced, lopinavir-naïve TITAN patient population, higher response rates were observed at 48 weeks in the darunavir/r arm compared with the lopinavir/ $\mathrm{r}$ arm, regardless of the number of darunavir RAMs or lopinavir RAMs at baseline. Virological response to darunavir/r was correlated with the number of darunavir RAMs at baseline, confirming the results of the POWER trial analysis. The best virological responses (VL $<50$ or $<400$ copies/mL) were seen in patients with 0-1 darunavir RAMs (De Meyer et al 2006, 2007). Safety data were similar between the groups; grade 3 or 4 adverse events occurred in 80 (27\%) of darunavir/r and $89(30 \%)$ lopinavir/r patients.

\section{Treatment-naïve individuals}

The randomized, phase III ARTEMIS study was conducted in 689 treatment-naïve subjects from 26 countries, and evaluated darunavir/r at a dose of $800 / 100 \mathrm{mg}$ once daily vs lopinavir/r either 400/100 mg bid or 800/200 mg once daily, with tenofovir and emtricitabine. This patient population was predominantly male $(70 \%)$, with a median CD4 count of 228 cells $/ \mathrm{mm}^{3}$ and a median viral load of 70,800 copies $/ \mathrm{mL}$. This study showed non-inferiority of darunavir/r compared with lopinavir/r, with $84 \%$ achieving viral load $<50$ copies $/ \mathrm{mL}$ vs $78 \%$ in the lopinavir/r arm. In this trial, there was a $13 \%(95 \%$ CI $1 ; 24 ; p<0.05)$ better virological response in patients receiving darunavir/r vs lopinavir/r once daily (DeJesus et al 2007). Virological failure occurred in $10 \%$ of subjects in the darunavir/r arm vs $14 \%$ in the lopinavir/r arm. Among subjects with VF, only 1 patient, in the lopinavir/r arm, harbored a new PI resistance mutation, A71T, V77I. Three subjects (1 in the darunavir/arm and 2 in the lopinavir/r arm) developed the $\mathrm{M} 184 \mathrm{~V} / \mathrm{I}$. In this study, both regimens were well tolerated, with more diarrhea occurring in the lopinavir/r arm $(10 \%$ vs $4 \%)$ and more rash in the darunavir/r arm (3\% vs $1 \%)$ (Clumeck et al 2007). Darunavir/r is not presently licensed for the treatment of HIV-1 in treatment-naïve individuals.

\section{Darunavir resistance profile}

In these pivotal clinical trials baseline darunavir susceptibility was predictive of virological response. Several studies have looked at the prevalence of darunavir-associated mutations in various populations. Table 1 summarizes the mutations associated with darunavir and tipranavir resistance. To estimate to what extent darunavir might be effective in patients failing distinct PIs in a clinical setting, the genotypic resistance scores for darunavir were examined in a large clinical HIV-1 drug resistance database. All clinical specimens from $\mathrm{HIV}$-infected patients failing PI-based regimens referred for drug resistance testing between 1999 and 2007 to a reference centre in Madrid were analyzed. A total of 1021 genotypes from patients failing lopinavir (39.2\%), nelfinavir (28.1\%), saquinavir (14.5\%), indinavir (13.7\%), atazanavir $(6.6 \%)$, fosamprenavir $(5.3 \%)$, and tipranavir $(1.1 \%)$ were identified. The prevalence of major darunavir resistance mutations was: I50V 2.1\%, I54M 1.3\%, L76V 2.7\%, and I84V 14.5\%. For minor darunavir resistance mutations, the rates were: V11I 3.3\%, V32I 3.9\%, L33F 11\%, I47V 2.1\%, I54L 2.3\%, G73S $12.8 \%$, and L89V 2.4\%. Overall, 6.7\% $(n=68)$ of the genotypes had 3 or more darunavir resistance mutations, which corresponded to a mean total number of PI resistance mutations of $12.3 \pm 1.9$. In the multivariate analysis, prior fosamprenavir failure, prior saquinavir failure, the total number of PI resistance mutations, and the number of prior PIs used were all independently associated with having more darunavir resistance mutations (Poveda et al 2007). In another study of treatment-experienced individuals, patients harboring viruses with amprenavir-specific resistance profiles, such as I50V or V32I + I47V, failed on darunavir/ r-containing regimens. These key amprenavir mutations were also selected at the time of failure, suggesting their impact on darunavir efficacy (Delaugerre et al 2007). Picchio and colleagues predicted phenotypic sensitivity to darunavir, 
Table I Resistance mutations associated with darunavir and tipranavir

\begin{tabular}{|c|c|c|c|c|c|c|c|c|c|c|c|c|c|c|c|}
\hline Protease codons & 10 & & 20 & 30 & & 40 & & & 50 & 60 & 70 & & & 80 & 90 \\
\hline & V & & & V L & & & & & I I I & & G & L & I & & $\mathrm{L}$ \\
\hline \multirow[t]{4}{*}{ Darunavir } & II & & & 3233 & & & & & 475054 & & 73 & 76 & 84 & & 89 \\
\hline & I & & & I F & & & & & $\vee \vee M$ & & S & $\mathrm{V}$ & $\mathrm{V}$ & & $\mathrm{V}$ \\
\hline & & & & & & & & & $\mathrm{L}$ & & & & & & \\
\hline & L & 1 & $\mathrm{~K}$ & L E & M & $\mathrm{K}$ & M & 1 & I & Q & $\mathrm{H}$ & $\mathrm{T}$ & V & $\mathrm{NI}$ & $\mathrm{L}$ \\
\hline \multirow[t]{4}{*}{ Tirpranavir } & 10 & 13 & 20 & 3335 & 36 & 43 & 46 & 47 & 54 & 58 & 69 & 74 & 82 & 8384 & 90 \\
\hline & V & V & $M$ & F G & I & $\mathrm{T}$ & $\mathrm{L}$ & V & A & E & $\mathrm{K}$ & $P$ & L & $\mathrm{D} \vee$ & $M$ \\
\hline & & & $\mathrm{R}$ & & & & & & M & & & & $\mathrm{T}$ & & \\
\hline & & & & & & & & & V & & & & & & \\
\hline
\end{tabular}

using over 56,000 sample genotypes with different levels of PI resistance, from the Virco database from 2004-5 (Picchio et al 2006; Vermeiren et al 2007). Clinical and/or biological cut-offs using upper and lower levels for each PI (3.4 and 99.6 for darunavir) were used to determine the relative sensitivity to darunavir, defined as maximal, reduced, and minimal sensitivity. Darunavir showed a low proportion of samples $(<5 \%)$ with minimal and reduced responses. In a subgroup $(\mathrm{n}=371)$ with minimal and reduced response to all PIs except darunavir and tipranavir, $\sim 70 \%$ had minimal or reduced response to both PIs, $\sim 20 \%$ had minimal or reduced response only to tipranavir with maximal response to darunavir, and $8 \%$ had minimal or reduced response to darunavir and maximal response to tipranavir.

Possible cross-resistance between tipranavir and darunavir has been further analyzed. Baseline isolates for 1468 patients from the RESIST trial and post-rebound isolates from 50 tipranavir-treated patients were sequenced and analyzed for Virtual Phenotype predicted IC50 fold change. For evaluation of cross-resistance, the clinical cut-offs of 1.2 and 5.4 for tipranavir and 3.4 and 96.9 for darunavir were used. From the entire tipranavir database, baseline and ontreatment genotypes were available and were analyzed for 278 patients. It was found that mutations at $47 \mathrm{~V}, 54 \mathrm{M}, 85 \mathrm{~V}$, and $73 \mathrm{~T}$ were most prevalent in isolates resistant to both PIs. Mutations 48V, 50V, and 54L were associated with resistance to darunavir but not to tipranavir. $82 \mathrm{~S}$ and $82 \mathrm{~T}$ were associated with resistance to tipranavir but not to darunavir. $30 \mathrm{~N}$ was associated with susceptibility to both drugs.

In patients from the RESIST trial, with baseline and on-treatment results, baseline isolates had median FC for tipranavir and darunavir of 1.6 and 3.55 respectively. After tipranavir treatment, medians were 8.4 and 2.5 , respectively. For the $80 \%(n=40)$ of on-treatment isolates with tipranavir above the upper clinical cut-off, 55\% were below the darunavir lower clinical cut-off. Loss of $48 \mathrm{MAV}, 50 \mathrm{~V}$, $54 \mathrm{ML}$, or $76 \mathrm{~V}$ and emergence of 34D were associated with declining darunavir FC. Selection for $82 \mathrm{~T} / \mathrm{L}$ was not associated with increased darunavir FC. In the larger tipranavir development program patient group $(n=278)$ the $82 \mathrm{~T} / \mathrm{L}$ mutation was the primary emergent mutation and there was a net loss of mutations at 48,50, and 76. Mutations 20R, $82 \mathrm{~T}$, and $82 \mathrm{~S}$ are only at increased prevalence in isolates resistant to tipranavir and susceptible to darunavir. Mutations at $46 \mathrm{I}, 10 \mathrm{~F}, 13 \mathrm{~V}, 32 \mathrm{I}, 33 \mathrm{~F}$, and $89 \mathrm{~V}$ are prevalent in both cross resistant isolates and darunavir resistant/tipranavir susceptible isolates. Mutations at $50 \mathrm{~V}$ and $54 \mathrm{~L}$ are prevalent only in darunavir resistant/tipranavir susceptible isolates. For mutations $54 \mathrm{M}, 73 \mathrm{~T}, 74 \mathrm{P}$, or $85 \mathrm{~V}$ nearly half of specimens were resistant to both tipranavir and darunavir. More than half of specimens with mutations $82 \mathrm{~F}, 82 \mathrm{~S}$, or $82 \mathrm{~T}$ were resistant to tipranavir and susceptible to darunavir. Almost $80 \%$ of specimens with $50 \mathrm{~V}$ mutation were resistant to darunavir and susceptible to tipranavir. More than half of specimens with mutations $20 \mathrm{~V}, 32 \mathrm{I}$, or $89 \mathrm{~V}$ were resistant to both tipranavir and darunavir. Almost $85 \%$ of specimens with the $30 \mathrm{~N}$ mutations were susceptible to both tipranavir and darunavir (Hall et al 2007).

\section{Conclusion}

Darunavir/ $\mathrm{r}$ is presently approved for use in treatmentexperienced HIV-1 infected patients. In this population, it is recommended that 2 or more active agents be used when possible. These can be new agents from existing classes such as the new NNRTI etravirine (Lazzarin et al 2007; Madruga et al 2007b). Other agents from new classes such as integrase inhibitors or CCR5 inhibitors have shown substantial virological and immunological efficacy, and could also be used in combination with darunavir/r in this patient population (Carter and Keating 2007; Grinsztejn et al 2007; Stephenson 2007). 
The integrase inhibitor raltegravir, and the CCR5 inhibitor maraviroc, have both been approved for use in treatmentexperienced patients (Groeschen 2007; Traynor 2007).

Darunavir/ $\mathrm{r}$ is an important addition to our treatment armamentarium in highly treatment-experienced individuals. It can provide a sustainable viral suppression in such patients, especially when combined with other active agents. Its high genetic barrier and its genetic resistance profile make it extremely useful in patients having failed a protease inhibitor containing regimen. The darunavir mutation score is distinct from the tipranavir mutation score, and this difference offers the clinician complementary options for subjects failing one or the other drug. In a modestly treatment-experienced population, darunavir/r can be a valuable option, particularly in subjects whose virus is resistant to lopinavir/r. Factors such as its strong genetic barrier for resistance development, relative pricing, and the available companion drugs to optimize the regimen backbone will need to be considered when contemplating its use in early treatment failure or in treatment-naïve populations.

\section{Disclosures}

The author has received consultant and/or speaker honoraria from Tibotec, Boehringer-Ingelheim, Merck, Pfizer, GlaxoSmith-Kline, Roche, Schering, Gilead, Virco, Virochem, Bayer Diagnostics, and Abbott.

\section{References}

Arasteh K, Clumeck N, Pozniak A, et al. 2005. TMC114/ritonavir substitution for protease inhibitor(s) in a non-suppressive antiretroviral regimen: a 14-day proof-of-principle trial. Aids, 19:943-7.

Busse KH, Penzak SR. 2007. Darunavir: A second-generation protease inhibitor. Am J Health Syst Pharm, 64:1593-602.

Carter NJ, Keating GM. 2007. Maraviroc. Drugs, 67, 2277-88; discussion 2289-90.

Clotet B, Bellos N, Molina JM, et al. 2007. Efficacy and safety of darunavir-ritonavir at week 48 in treatment-experienced patients with HIV-1 infection in POWER 1 and 2: a pooled subgroup analysis of data from two randomised trials. Lancet, 369:1169-78.

Clumeck N, Chiliade P, Clotet B, et al. 2007. ARTEMIS - Efficacy and Safety of Lopinavir (BID vs QD) and Darunavir (QD) in AntiretroviralNaive Patients 11th European AIDS Conference. Madrid, Spain.

De Meyer S, Azijn H, Surleraux D, et al. 2005. TMC114, a novel human immunodeficiency virus type 1 protease inhibitor active against protease inhibitor-resistant viruses, including a broad range of clinical isolates. Antimicrob Agents Chemother, 49:2314-21.

De Meyer S, Lefebvre E, Azijn H, et al. 2006. Phenotypic and genotypic determinants of resistance to TMC114: pooled analysis of POWER 1, 2 and 3. 15th International HIV Drug Resistance Workshop. Sitges, Spain.

De Meyer S, Vangeneugden T, van Baelen B, et al. 2007. Effect of baseline and on-treatment mutations on the antiretroviral activity of darunavir/ ritonavir (DRV/r) and lopinavir/ritonavir (LPV/r): results of a randomised, controlled, phase III study (TITAN). 4th International AIDS Society Conference on HIV Pathogenesis, Treatment and Prevention. Sydney, Australia.

De Meyer S, Vangeneugden T, Van Baelen B, et al. 2008. Resistance profile of darunavir: combined 24-week results from the POWER trials. AIDS Res Hum Retroviruses, 24:379-88.
Dejesus E, Ortiz R, Khanlou H. 2007. Efficacy and safety of darunavir/ ritonavir versus lopinavir/ritonavir in ARV treatment-naive HIV-1infected patients at week 48: ARTEMIS. 47th Annual Interscience Conference on Antimicrobial Agents and Chemotherapy (ICAAC). Chicago, Illinois.

Delaugerre C, Mathez D, Peytavin G, et al. 2007. Key amprenavir resistance mutations counteract dramatic efficacy of darunavir in highly experienced patients. Aids, 21:1210-3.

[DHHS] US Department of Health and Human Services. 2008. Guidelines for the use of antiretroviral agents in HIV-1-infected adults and adolescents. In DHHS (Ed.).

Flexner C. 1998. HIV-protease inhibitors. N Engl J Med, 338:1281-92.

Ghosh AK, Chapsal BD, Weber IT, et al. 2007. Design of HIV protease inhibitors targeting protein backbone: an effective strategy for combating drug resistance. Acc Chem Res, 41:78-86.

Ghosh A K, Sridhar PR, Leshchenko S, et al. 2006. Structure-based design of novel HIV-1 protease inhibitors to combat drug resistance. $J$ Med Chem, 49:5252-61.

Grinsztejn B, Nguyen BY, Katlama C, et al. 2007. Safety and efficacy of the HIV-1 integrase inhibitor raltegravir (MK-0518) in treatmentexperienced patients with multidrug-resistant virus: a phase II randomised controlled trial. Lancet, 369:1261-9.

Groeschen HM. 2007. Novel HIV treatment approved. Am J Health Syst Pharm, 64:1886.

Hall DB, Schapiro J, Boucher CAB, et al. 2007. Genotypic relationships between tipranavir and darunavir resistance in protease inhibitor experienced patients. 6th Intl HIV Drug Resistance Workshop. Barbados, West Indies.

Hammer SM, Saag MS, Schechter M, et al. 2006. Treatment for adult HIV infection: 2006 recommendations of the International AIDS Society - USA panel. Top HIV Med, 14:827-43.

Johnson VA, Brun-Vezinet F, Clotet B, et al. 2007. Update of the drug resistance mutations in HIV-1: 2007. Top HIV Med, 15:119-25.

Katlama C, Esposito R, Gatell JM, et al. 2007. Efficacy and safety of TMC114/ritonavir in treatment-experienced HIV patients: 24-week results of POWER 1. Aids, 21:395-402.

King NM, Prabu-Jeyabalan M, Nalivaika EA, et al. 2004. Structural and thermodynamic basis for the binding of TMC114, a next-generation human immunodeficiency virus type 1 protease inhibitor. $J$ Virol, 78:12012-21.

Koh Y, Matsumi S, Das D, et al. 2007. Potent inhibition of HIV-1 replication by novel non-peptidyl small molecule inhibitors of protease dimerization. J Biol Chem, 39:28709-20.

Koh Y, Nakata H, Maeda K, et al. 2003. Novel bis-tetrahydrofuranylurethanecontaining nonpeptidic protease inhibitor (PI) UIC-94017 (TMC114) with potent activity against multi-PI-resistant human immunodeficiency virus in vitro. Antimicrob Agents Chemother, 47:3123-9.

Kovalevsky AY, Liu F, Leshchenko S, et al. 2006a. Ultra-high resolution crystal structure of HIV-1 protease mutant reveals two binding sites for clinical inhibitor TMC114. J Mol Biol, 363:161-73.

Kovalevsky AY, Tie Y, Liu F, et al. 2006b. Effectiveness of nonpeptide clinical inhibitor TMC-114 on HIV-1 protease with highly drug resistant mutations D30N, I50V, and L90M. J Med Chem, 49:1379-87.

Kuritzkes DR. 2007. HIV resistance: frequency, testing, mechanisms. Top HIV Med, 15:150-4.

Lazzarin A, Campbell T, Clotet B, et al. 2007. Efficacy and safety of TMC125 (etravirine) in treatment-experienced HIV-1-infected patients in DUET-2: 24-week results from a randomised, double-blind, placebocontrolled trial. Lancet, 370:39-48.

Madruga JV, Berger D, McMurchie M, et al. 2007a. Efficacy and safety of darunavir-ritonavir compared with that of lopinavir-ritonavir at 48 weeks in treatment-experienced, HIV-infected patients in TITAN: a randomised controlled phase III trial. Lancet, 370:49-58.

Madruga JV, Cahn P, Grinsztejn B, et al. 2007b. Efficacy and safety of TMC125 (etravirine) in treatment-experienced HIV-1-infected patients in DUET-1: 24-week results from a randomised, double-blind, placebocontrolled trial. Lancet, 370:29-38. 
Mascolini M, Boucher C, Larder B, et al. 2007. Key reports from the XV International HIV Drug Resistance Workshop 2006. Antivir Ther, 12:131-45.

Molina JM, Cohen C, Katlama C, et al. 2007. Safety and efficacy of darunavir (TMC114) with low-dose ritonavir in treatment-experienced patients: 24-week results of POWER 3.J Acquir Immune Defic Syndr, 46:24-31.

Picchio G. 2006. Analyses of susceptibility and cross-resistance between TMC114 and other protease inhibitors among $>56,000$ routine samples, using linear regression model-based fold change predictions. XV International Drug Resistance Workshop. Sitges, Spain.

Poveda E, de Mendoza C, Martin-Carbonero L, et al. 2007. Prevalence of darunavir resistance mutations in HIV-1-infected patients failing other protease inhibitors. J Antimicrob Chemother, 60:885-8.

Stephenson J 2007. Researchers buoyed by novel HIV drugs: will expand drug arsenal against resistant virus. JAMA, 297:1535-6.
Surleraux DL, Tahri A, Verschueren WG, et al. 2005. Discovery and selection of TMC114, a next generation HIV-1 protease inhibitor. $J$ Med Chem, 48:1813-22.

Tie Y, Kovalevsky AY, Boross P, et al. 2007. Atomic resolution crystal structures of HIV-1 protease and mutants V82A and I84V with saquinavir. Proteins, 67:232-42.

Traynor K. 2007. Integrase inhibitor gains FDA approval. Am J Health Syst Pharm, 64:2310.

Vangeneugden. 2006. Impact of optimised background regimen on virological response to TMC114 with low-dose ritonavir in POWER 1, 2 and 3, as measured by the phenotypic susceptibility score. 15th International HIV Drug Resistance Workshop. Sitges, Spain.

Vermeiren H, van Craenenbroeck E, Alen P, et al. 2007. Prediction of HIV1 drug susceptibility phenotype from the viral genotype using linear regression modeling. J Virol Methods, 145:47-55. 
